corticosteroid use have been described as cardiovascular risk factors but there is controversy surrounding antimalarials as a protective factor. Our objective is to analyze associated factors with the presentation of cardiovascular events such as high blood pressure (HBP), acute myocardial infarction (AMI), stroke and thromboembolic disease (TED)

Methods A cross-sectional study was done with 1175 records of patients with SLE that fulfilled either ACR 1997 of SLICC 2012 classification criteria that had been in medical care between 2015 and 2017 in a rheumatology specialized institution in six cities of Colombia. We describe sociodemographic, clinical and immunoserological characteristics and a comparative analysis was done with chi2 and Mann Whitney's U with a combined outcome of cardiovascular disease obtaining an OR of crude associations that were adjusted for several variables

Results Women represented $91 \%$ of the cohort with a median age of 44 years (IQR 21) and 8 years of disease duration (IQR 11) with a mean age at diagnosis of 32 years, 5,4\% were active smokers and $15 \%$ had smoked in the past. Cardiovascular events were found in $32 \%$ of the patients with HT as the most common. Other cardiovascular outcomes such as stroke, TED and AMI were infrequent with a prevalence of $3.3 \%, 2.9 \%$ and $2 \%$ respectively. In bivariate analysis, age $>36$ years and corticosteroid use were associated with a significantly higher risk, while the use of antimalarials for more than 6 years was found to protect for cardiovascular risk with no difference between chloroquine and hydroxychloroquine use (table 1)

Conclusions Our cohort is comparable with other SLE cohorts regarding the frequency of cardiovascular events. Up to $32 \%$ of the described population presented a cardiovascular event and arterial hypertension was the most frequent. Continuous use of antimalarials for more than 6 years has a protective effect against cardiovascular events such as arterial hypertension, stroke, acute myocardial infarction and thromboembolic disease. The benefit seen only after 6 years of continuous use probably reflects the need of a long period of time before some of the potential benefits of these medications are seen Funding Source(s): None

\section{INCREASED MORTALITY AMONG PATIENTS WITH SYSTEMIC LUPUS ERYTHEMATOSUS AFTER HYDROXYCHLOROQUINE DISCONTINUATION}

${ }^{1}$ Antonio Avina-Zubieta*, ${ }^{2}$ April Jorge, ${ }^{3}$ Mary A DeVera, ${ }^{4} \mathrm{Na} \mathrm{Lu},{ }^{4}$ John Esdaile, ${ }^{5}$ Hyon Choi. ${ }^{1}$ Arthritis Research Canada, Univeristy of British Columbia; ${ }^{2}$ Division of Rheumatology, Allergy, and Immunology, Massachusetts General Hospital; ${ }^{3}$ University of British Columbia; ${ }^{4}$ Arthritis Research Canada; ${ }^{5}$ Massachusetts General Hospital

\subsection{6/lupus-2019-Ism.299}

Background Hydroxychloroquine (HCQ) is near-universally recommended for patients with SLE. Use of this medication has previously been associated with a substantial survival benefit among SLE patients. We aimed to determine the potential temporal association between HCQ discontinuation and allcause and cardiovascular disease (CVD) mortality.

Methods We conducted a population-based case-control study using an administrative health database including the entire population in the province of British Columbia, Canada (>5 million individuals). We identified cases with SLE who died and each case was matched on age, sex, and SLE disease duration with living controls with SLE. We used conditional logistic regression to assess the association between current use of HCQ or recent discontinuation of HCQ and the risk of all-cause and cause-specific mortality relative to remote HCQ users. Remote users were defined by a duration greater than 365 days between the last HCQ prescription and the index date (i.e., death date). Recent users had a duration less than 365 days since the last HCQ prescription and index date. Current users had active HCQ prescriptions spanning

Abstract 299 Table 1 Risk of Death with Current Usage, Non-Usage, and Recent Discontinuation compared with Remote Usage of Hydroxychloroquine among patients with Systemic Lupus Erythematosus

\begin{tabular}{|l|c|c|c|c|}
\hline All-Cause Mortality & $\begin{array}{c}\text { Cases, } \\
\text { N }\end{array}$ & $\begin{array}{c}\text { Controls, } \\
\mathbf{N}\end{array}$ & $\begin{array}{c}\text { Crude Odds } \\
\text { Ratio (95\% CI) }\end{array}$ & $\begin{array}{c}\text { Adjusted Odds } \\
\text { Ratio (95\% CI) }\end{array}$ \\
\hline Remote HCQ Users & 72 & 106 & 1.0 (reference) & 1.0 (reference) \\
\hline Recent HCQ Discontinuers & 65 & 34 & $3.03(1.77-5.17)$ & $3.78(2.07-6.91)$ \\
\hline Current HCQ users & 32 & 156 & $0.30(0.18-0.49)$ & $0.35(0.20-0.59)$ \\
\hline HCQ Non-users & 121 & 206 & $0.83(0.55-1.24)$ & $0.93(0.59-1.44)$ \\
\hline $\begin{array}{l}\text { Cardiovascular Disease } \\
\text { Mortality }\end{array}$ & & & & \\
\hline Remote HCQ Users & 17 & 28 & $1.0($ reference) & 1.0 (reference) \\
\hline Recent HCQ Discontinuers & 17 & 11 & $2.57(0.96-6.92)$ & $4.63(1.31-16.42)$ \\
\hline Current HCQ users & 9 & 44 & $0.32(0.12-0.87)$ & $0.37(0.11-1.27)$ \\
\hline HCQ Non-users & 40 & 59 & $1.11(0.51-2.44)$ & $1.15(0.45-2.99)$ \\
\hline Other Cause Mortality & & & & \\
\hline Remote HCQ Users & 35 & 52 & $1.0($ reference) & $1.0($ reference) \\
\hline Recent HCQ Discontinuers & 33 & 18 & $3.17(1.45-6.93)$ & $3.90(1.56-9.75)$ \\
\hline Current HCQ users & 13 & 72 & $0.28(0.14-0.58)$ & $0.32(0.14-0.71)$ \\
\hline HCQ Non-users & 57 & 95 & $0.89(0.49-1.64)$ & $1.18(0.29-2.37)$ \\
\hline
\end{tabular}


the index date. Fully adjusted models included chronic kidney disease, Charlson comorbidity index, glucocorticoids, and cardiovascular medication use assessed at the time of SLE diagnosis.

Results We identified 290 SLE cases who died and 502 matched controls among 792 individuals with SLE. The mean age at index date was 65.6 years for cases and 64.7 years for controls. The majority were female $(87.9 \%$ of cases and 91.4\% of controls). The mean SLE disease duration was 5.3 years for both groups. Adjusted odd ratios (ORs) for all-cause mortality relative to the remote users were 0.35 (95\% CI: $0.20,0.59$ ) for current users and 3.78 (95\% CI: 2.07, 6.91) for subjects who recently discontinued HCQ (table 1). HCQ non-users had the same risk of death as remote users (OR 0.93 [95\% CI: $0.59,1.44])$. Similar trends were seen for the risk of mortality due to CVD.

Conclusions In this study, we found a nearly four-fold increased risk of death associated with recent HCQ discontinuation and a substantially increased risk of CVD death. This could be partially explained by a direct protective effect of HCQ that is rapidly lost following discontinuation. We also demonstrated a $65 \%$ reduced risk of death among current HCQ users compared with remote users. By leveraging remote

users as the comparison group, we reduced the potential for confounding by indication.

Funding Source(s): CIHR (Grants MOP 125960 and THC 135235).

\section{INSIGHTS FROM SINGLE-CELL RNA SEQUENCING OF SKIN AND KIDNEY IN LUPUS NEPHRITIS}

${ }^{1}$ Evan Der*, ${ }^{2}$ Hemant Suryawanshi, ${ }^{2}$ Pavel Morozov, ${ }^{2}$ Manjunath Kustagi, ${ }^{3}$ Beatrice Goilav, ${ }^{3}$ Saritha Ranabothu, ${ }^{4}$ Peter Izmirly, ${ }^{4}$ Robert Clancy, ${ }^{4}$ Michael Belmont, ${ }^{5}$ Mordecai Koenigsberg, ${ }^{1}$ Michele Mokrzycki, ${ }^{5}$ Helen Rominiecki, ${ }^{5}$ Jay Graham, ${ }^{5}$ Juan Rocca, ${ }^{4}$ Nicole Bornkamp, ${ }^{1}$ Nicole Jordan, ${ }^{1}$ Emma Schulte, ${ }^{4}$ Ming Wu, ${ }^{5}$ James Pullman, ${ }^{6}$ Kamil Slowikowski, ${ }^{6}$ Soumya Raychaudhuri, ${ }^{7}$ Joel Guthridge, ${ }^{7}$ Judith A James, ${ }^{4}$ Jill Buyon, ${ }^{2}$ Thomas Tuschl, ${ }^{8}$ Chaim Putterman. ${ }^{1}$ Albert Einstein College of Medicine; ${ }^{2}$ The Rockefeller University; ${ }^{3}$ Children's Hospital at Montefiore; ${ }^{4}$ NYU School of Medicine; ${ }^{5}$ Montefiore Medical Center; ${ }^{6}$ Broad Institute; ${ }^{7}$ Oklahoma Medical Research Foundation; ${ }^{8}$ Albert Einstein College of Medicine and Montefiore Medical Center

\subsection{6/lupus-2019-Ism.300}

Background Classification and treatment decisions in lupus nephritis (LN) are largely based on renal histology. Single-cell

C

A

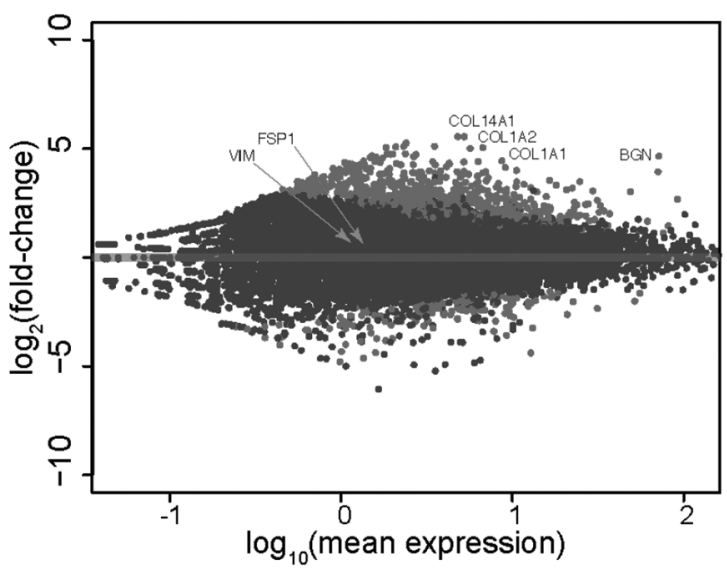

B

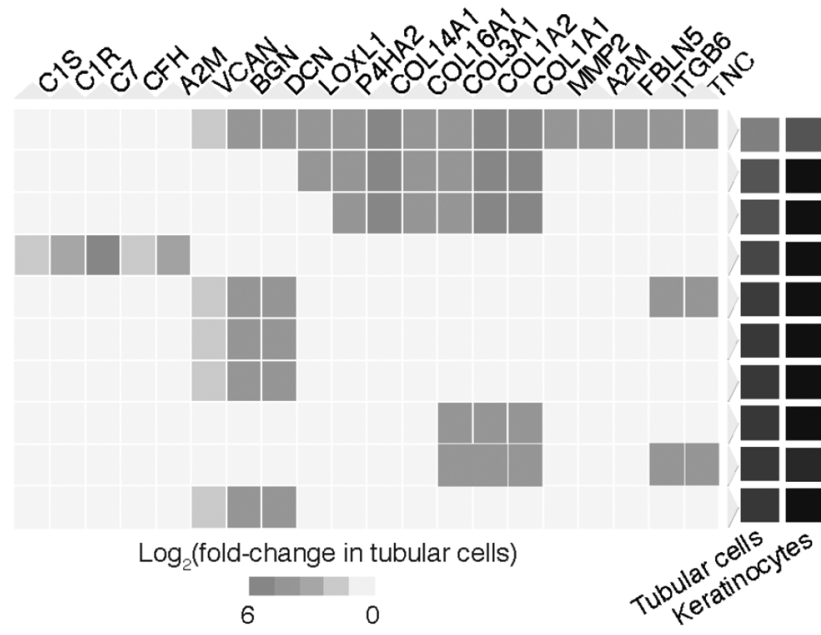

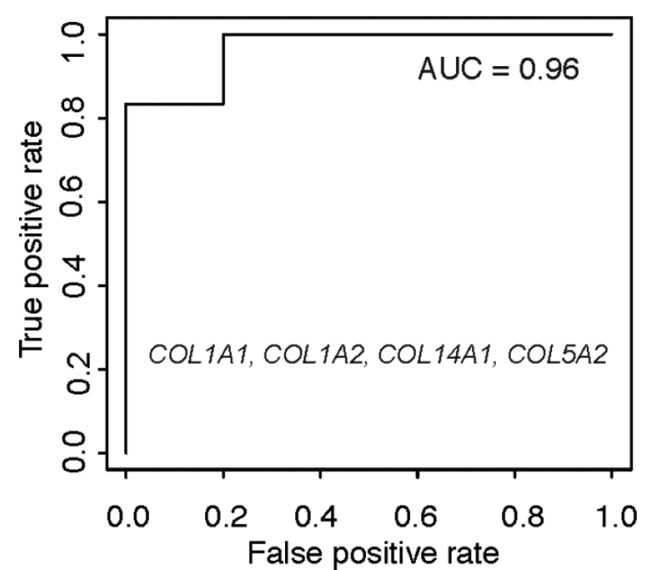

\section{Enriched Terms}

Extracellular matrix organization

Collagen formation

Collagen biosynthesis and modifying enzymes

Complement and coagulation cascades

ECM proteoglycans

CSIDS degradation

Dermatan sulfate biosynthesis

Scavenging by Class A Receptors

ECM-receptor interaction

Defective CHST3 causes SEDCJD

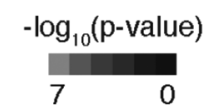

Abstract 300 Figure 1 A fibrotic gene signature as a potential prognostic marker for patients non-responsive to treatment. A) MA plot of differential expression analysis performed between tubular cells of patients responsive $(n=13)$ or non-responsive to treatment $(n=5)$. Significantly differentially expressed genes are colored in red. B) Pathway enrichment analysis of genes identified as upregulated in patients non-responsive to treatment. -Log10(p-value) of each pathway is shown for both keratinocytes and tubular cells colored from least significant (black) to most significant (red). Log2 fold change in gene expression between patients non-responsive to treatment compared with patients responsive to treatment in each pathway is indicated for tubular cells from smallest (grey) to highest (orange). C) Receiver operating characteristic curve of the logistic regression equation of differentially expressed fibrotic genes, COL1A2, COL1A1, COL14A1, COL5A2, with area under the curve (AUC) indicated. 